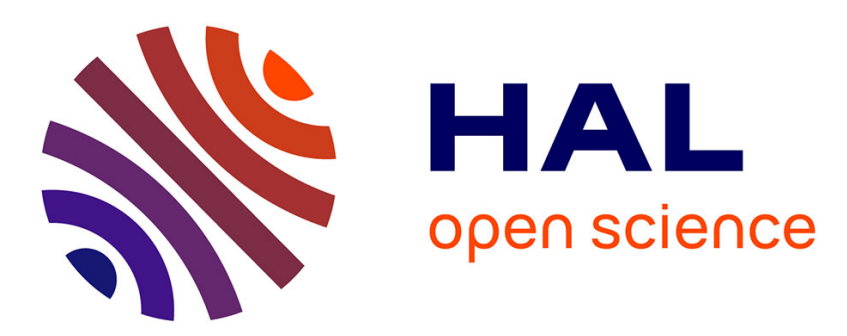

\title{
TRANSMISSION DE L'HISTOIRE FAMILIALE ET DE LA MÉMOIRE HISTORIQUE FACE À LA PRÉCARITÉ
}

Catherine Delcroix

\section{- To cite this version:}

Catherine Delcroix. TRANSMISSION DE L'HISTOIRE FAMILIALE ET DE LA MÉMOIRE HISTORIQUE FACE À LA PRÉCARITÉ. Migrations Société, 2009, Transmissions familiales en migration, 21 (123-124), pp.143-157. hal-01284366

\section{HAL Id: hal-01284366 https://hal.science/hal-01284366}

Submitted on 9 Mar 2016

HAL is a multi-disciplinary open access archive for the deposit and dissemination of scientific research documents, whether they are published or not. The documents may come from teaching and research institutions in France or abroad, or from public or private research centers.
L'archive ouverte pluridisciplinaire HAL, est destinée au dépôt et à la diffusion de documents scientifiques de niveau recherche, publiés ou non, émanant des établissements d'enseignement et de recherche français ou étrangers, des laboratoires publics ou privés. 


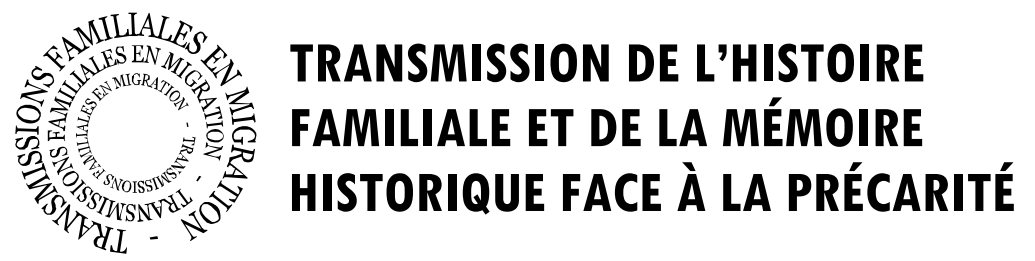

Catherine DELCROIX *

Depuis la fin des années 1980 nous nous sommes penchée sur la manière dont les familles ouvrières originaires du Maghreb éduquent leurs enfants tout en faisant face aux difficultés liées à la précarité : chômage, manque chronique d'argent, discriminations... ${ }^{1}$ C'est à partir des résultats de ces travaux que dans cette contribution nous nous proposons de décrire des stratégies éducatives de parents fondées sur le travail de transmission intergénérationnelle susceptibles d'aider leurs enfants à prévenir les risques liés à la précarité et accentués par le vécu de la discrimination.

Pour étudier ces stratégies, nous avons mis en place une démarche méthodologique fondée sur la reconstruction d'historiques de familles, reconstitués à partir du recueil d'entretiens auprès de plusieurs membres d'une même famille (parents, enfants). Nous avons multiplié les études de cas dans différentes régions et cités HLM de France. Pour chacune des enquêtes, le choix des familles s'est fondé sur les mêmes critères : interroger des personnes qui vivent des configurations semblables et qui sont confrontées aux mêmes types de problèmes sur le plan migratoire, professionnel, économique et familial. II s'agissait d'identifier les différents types de parcours permettant de montrer la diversité des profils des habitants vivant dans ces cités.

Ces familles immigrées, appartenant aux milieux populaires, sont souvent considérées comme dépourvues de ressources. Elles sont même

Professeur de sociologie, Laboratoire Cultures et Sociétés en Europe, Université de Strasbourg, Maison interdisciplinaire des sciences de l'homme d'Alsace (MISHA), Strasbourg.

1. Cf. DELCROIX, Catherine, "Les parents des cités : la prévention familiale des risques encourus par les enfants", Annales de la Recherche Urbaine, n 83-84, 1999, pp. 97-107 ; DELCROIX, Catherine, "La complexité des rapports intergénérationnels dans les familles ouvrières du Maghreb. L'exemple de la diagonale des générations", Temporalités, $n^{\circ}$ 2, 2004, pp. 44-59. 
stigmatisées et confrontées à des préjugés tenaces : elles seraient responsables de leurs difficultés, incapables de gérer un budget, de discipliner leurs enfants et de les aider à s'insérer dans la société... Pourtant, les parcours de réussite de certains parents et enfants de ces milieux sont a priori jugés improbables. On examinera ici les ressources subjectives que ces personnes, souvent dépourvues de capital économique, culturel et social, utilisent pour y parvenir. II faut entendre par ressources subjectives les énergies physiques, mentales et morales qu'un individu possède et développe à un moment donné de son existence, ainsi que les savoirs et savoir-faire qui lui permettent de mobiliser à bon escient ces énergies, voire celles de ses proches, pour répondre aux besoins et réaliser les projets familiaux et personnels. II s'agit aussi de ce qu'Antony Giddens définit comme des " compétences pratiques et des compétences discursives $11^{2}$. Or, ces ressources subjectives sont construites et mobilisées dans le cadre de processus de transmissions intergénérationnelles et intragénérationnelles.

Pour mieux saisir la dynamique complexe des rapports entre générations, rapports de genre et systèmes d'action au sein de ces familles, nous avons pris la décision d'en suivre une sur plusieurs années. La situation de la famille Nour ${ }^{3}$ nous a paru cristalliser de manière exemplaire les nombreux obstacles constitutifs de la précarité. Les parents Nour et chacun de leurs enfants y apportent des réponses différenciées. Tenaces, ils mettent en œuvre des stratégies qui sont à la fois originales et communes à bien d'autres familles.

Il s'agit d'une famille marocaine composée de huit enfants (deux filles et six garçons), habitant une cité HLM, plongée comme beaucoup d'autres dans des situations et des modes de vie qui reposent sur des équilibres instables et faisant partie du monde ouvrier. Ses revenus lui viennent d'un emploi de maçon que le père a exercé pendant 11 ans ; mais, victime d'un accident du travail, il ne perçoit depuis lors qu'une petite pension d'invalidité complétée par des allocations familiales. Ces moyens financiers sont tout juste suffisants pour assurer les besoins primaires du ménage, ce qui oblige à calculer au centime près. Cette situation les rend plus sensibles que d'autres aux perturbations de la vie.

2. GIDDENS, Antony, La constitution de la société. Éléments de la théorie de la structuration, Paris : Presses universitaires de France, 1987, $474 \mathrm{p}$.

3. Cf. DELCROIX, Catherine, Ombres et lumières de la famille Nour. Comment certains résistent face à la précarité, Paris : Éd. Payot, 2001, 258 p. (édition 2005 augmentée). Jusqu'à aujourd'hui, nous continuons à suivre la destinée de ses membres. 
Cette précarité influence le comportement des parents Nour et celvi de leurs huit enfants. Chacun de ces derniers a une trajectoire particulière, mais tous sont aussi membres d'une famille, elle-même membre d'un quartier qui fait partie d'une grande ville appartenant à la société française. Comment ces huit enfants issus du monde ouvrier, confrontés à la pauvreté, au racisme et à la disparition des emplois industriels construisent-ils leur avenir? Quels obstacles rencontrent-ils? Quelles situations amènent une partie d'entre eux à connaître des difficultés répétées d'insertion dans la société française? Pourquoi certains d'entre eux y répondent-ils par la violence destructrice — pour eux-mêmes et leur environnement - et d'autres par l'effort et l'imagination?

Depuis la naissance de leurs enfants (au moment de notre première rencontre, en 1997, l'aîné avait 25 ans et le benjamin 5 ans), les parents ont orienté leur projet migratoire vers la recherche d'une insertion satisfaisante de leurs deux filles et de leurs six fils dans la société française. Ils partagent cet objectif avec la plupart des familles qui ont émigré 4 . Conscients des risques d'échec et du rejet dont leurs enfants peuvent devenir des victimes, ils ont développé des stratégies éducatives susceptibles de leur donner confiance en eux et de les aider à franchir les obstacles, notamment en insistant sur l'importance de l'école, avec des résultats très contrastés d'un enfant à l'autre.

\section{La transmission de l'histoire familiale et ses effets sur les rapports des enfants à leur avenir dans la société française}

Les parents de la famille Nour sont illettrés. Comment, dans ce cas, alors qu'ils n'ont ni expérience scolaire ni connaissance du fonctionnement des institutions françaises peuvent-ils aider leurs enfants à s'adapter aux attentes de l'école et de la société françaises? En réfléchissant avec leurs enfants sur leur propre enfance et sur leurs modèles familiaux d'origine, ils ont mis sur pied une organisation familiale et un discours parental justifiant la nécessité de consacrer du temps et de l'énergie à l'école. II s'agit aussi pour eux de faire apparaître les possibilités que la société française leur propose afin que leurs enfants puissent les saisir.

4. Cf. ZEROULOU, Zahia, "La réussite scolaire des enfants d'immigrés. L'apport d'une approche en termes de mobilisation", Revue Française de Sociologie, vol. 29, $\mathrm{n}^{\circ} 3$, juillet-septembre 1988, pp. 447470 ; ZEHRAOUI, Ahsène, Familles d'origine algérienne en France. Étude sociologique des processus d'intégration, Paris : Éd. CIEMI - L'Harmattan, 1999, 310 p. 
À la base donc se trouve la transmission de la mémoire familiale associée à la volonté parentale de réussir le processus de socialisation de leurs enfants. Pour Robert Neuburger, cette transmission est II la capacité donnée à un sujet de disposer d'un quota de certitudes ou du moins d'informations sur ce qu'est une famille, sa famille, de telle sorte qu'il puisse articuler son propre projet fondateur, soit en continuité, soit en rupture avec la génération précédente : la transmission, c'est la transmission de la capacité de transmettre $1{ }^{5}$. La qualité de cette mémoire familiale se fonde, selon Toshiaki Kozakaii, sur le fait que ce qui nous lie à notre père et à notre mère n'est nullement un lien du sang biologique, mais la mémoire d'avoir partagé notre enfance et notre adolescence avec eux ${ }^{6}$. Encore faut-il trouver les modes adéquats de communication entre générations... Comme le souligne Jean Piaget dans son ouvrage Jugement moral chez l'enfant ${ }^{7}$, dans le processus de socialisation il ne faut pas négliger le rôle de l'activité des enfants eux-mêmes, leur rôle en tant qu'acteurs de leur propre socialisation. Cet auteur a défini la socialisation comme une double interaction (appelée " équilibration ") entre l'individu et son environnement social : à la fois "accommodation" (action sur soi pour s'adapter à l'environnement) et "assimilation" (action sur l'environnement pour l'adapter à soi), la socialisation n'est pas chez lui un équivalent de la scolarisation, mais une "I imprégnation informelle et implicite ". Les moments passés en famille doivent permettre de donner du sens aux expériences passées et présentes vécues par les parents et les enfants.

Une des questions qui préoccupent les enfants de la famille Nour porte sur l'histoire de leur père depuis l'enfance. Ils veulent comprendre ce qu'il a vécu, pourquoi et dans quelles conditions il est venu en France et ce qui l'a amené à y rester.

Amin, le père, est né en 1943 dans un village de l'Ouest marocain. II est l'aîné de 13 enfants. Très tôt il participe aux activités agricoles : " Depuis l'âge de 10 ans, i'ai fait berger pour les moutons et les vaches. Et à l'âge de 18 ans, i'ai fait l'agriculture. Et après je suis venu en France. D'autres gars étaient venus en France avant moi, alors moi aussi je suis venu 11.

Le récit qu'il fait par bribes de sa vie d'enfant puis d'adulte est toujours relié à l'obligation d'assurer la survie du groupe et à sa fierté

5. NEUBURGER, Robert, Le mythe familial, Paris : Éd. ESF, 1995, $180 \mathrm{p}$.

6. Cf. KOZAKAII, Toshiaki, L'étranger, l'identité. Essai sur l'intégration culturelle, Paris : Éd. Payot et Rivages, 2000, $227 \mathrm{p}$.

7. PIAGET, Jean, Jugement moral chez l'enfant, Paris: Presses universitaires de France, 2000

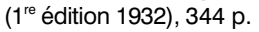


d'être pourvoyeur. L'entreprise familiale traditionnelle (une ferme) conduit tous les hommes de la famille à se penser comme coresponsables de sa gestion. Son économie se fonde sur sa capacité à produire de la nourriture pour tous, ce qui n'a pas été le cas chaque année.

En tant que frère aîné, Amin est considéré plus responsable que ses frères et très respecté. "L'un de ses frères [qui habite dans la même cité HLM en France] n'ose pas fumer devant lui II, rapporte sa fille Leila. Cette forme de socialisation a pour conséquence de faire passer l'intérêt du groupe avant celui des individus. Dans la famille d'Amin, comme dans d'autres, on retrouve des formes de solidarité familiales communes : envoi de mandats, hébergement de parents ou aide à la gestion de la vie pratique des parents âgés.

Ce sens de la famille et des responsabilités familiales, dont Amin a beaucoup parlé à ses enfants, est sans doute ce qui les a amenés à privilégier leur maintien prolongé au domicile familial ou à proximité, dans la même cité HLM, pour poursuivre les échanges et perpétuer la solidarité intragénérationnelle et intergénérationnelle. Face aux risques qu'implique la précarité, il n'y a pas eu transmission de la volonté d'utiliser la mobilité géographique pour s'en sortir, mais bien la transmission du besoin de l'entraide familiale. Cette attitude et ses pratiques ont parfois empêché certains enfants de la famille Nour de saisir des possibilités d'emploi qui les auraient éloignés de ce havre protecteur.

Pour Amin, l'apprentissage du combat contre la pauvreté s'est fait très jeune. La débrouillardise fait partie des valeurs qu'il enseigne à ses enfants. Il est très fier de sa connaissance des chevaux et des parures qu'il a réalisées au Maroc : " Comme lui-même nous l'a dit, raconte Leila, mais aussi ma grand-mère et mon grand-père, il était le seul à savoir monter aussi bien les chevaux. Une fois par an tous les habitants de son village se réunissaient pour organiser une foire de chevaux et une course. Dans chaque famille, un enfant mâle avait la responsabilité de décorer un cheval et de le monter pour défendre l'honneur familial. Les parures étaient très belles. Cet art se transmettait aux garçons de génération en génération 11.

Dans ce contexte rural marqué par la pauvreté, le talent du jeune garçon n'avait pas qu'une signification symbolique. Le prestige ainsi conféré à travers lui à tout son groupe familial avait des conséquences économiques. Sur le marché où son père et ses oncles vendaient leurs produits, on avait une raison supplémentaire de leur acheter leurs marchandises plutôt qu'à leurs concurrents. Habileté, sens artistique et effort étaient associés. 
Chaque soir, quand il allait endormir ses enfants, Monsieur Nour racontait cette histoire et aussi certaines expériences qui l'ont aidé à devenir un ouvrier courageux, reconnu comme un excellent maçon. II arrêtait son récit au moment où plusieurs accidents du travail, en 1982, ont interrompu de manière définitive son parcours professionnel. De ces moments douloureux, il refuse de parler : "Ie veux que mes enfants voient les bons côtés de la France; je ne veux pas qu'ils croient que tous les patrons sont des exploiteurs qui mettent en danger les ouvriers. Les enfants ont tendance à voir les mauvais côtés de la France et pas les avantages 11.

Amin a conscience que ses enfants, comme ceux de ses voisins, ont tendance à se révolter car ils pensent être traités injustement, comme des sous-citoyens. Par exemple, Djamel — qui à l'époque de l'entretien avait 21 ans - a répété à plusieurs reprises : "Au moment où mon père cherchait du travail il en trouvait facilement. C'était du travail pénible mais il en trouvait. Nous, on a été à l'école mais on ne veut pas de nous pour travailler ! C'est pas juste II. Mais son père ne veut pas accentuer ce sentiment d'injustice. Il considère d'abord que la situation qu'il a vécue au Maroc était plus difficile : il n'a pas pu aller à l'école, et même si en France il y a du racisme, les contraintes liées à la précarité n'y sont pas aussi importantes. II pense aussi que l'attitude de révolte empêche les jeunes de se saisir des occasions que la société française leur offre en termes d'accès aux études et aux droits sociaux (santé, chômage, retraite, aides ponctuelles).

Dans cette famille comme dans beaucoup d'autres, la principale difficulté rencontrée par les jeunes adultes porte sur la recherche d'emploi : " C'est vrai, explique Driss, que pendant toute notre enfance notre père a été super, il nous racontait des histoires sur le Maroc, sur quand il était berger et qu'il se levait trop tôt le matin pour sortir les bêtes. C'était toujours le travailleur. II donnait l'impression que le bon Dieu ne l'avait mis au monde que pour travailler. Et à partir du moment où on lui a enlevé ce travail, il n'était plus rien $1{ }^{8}$.

Les moments d'intimité se déroulent le soir et sont ancrés dans les souvenirs de ses enfants comme autant de signes de la valeur du travail et de l'engagement professionnel. Lorsque leur père est déclaré invalide et qu'il ne peut plus travailler, il descend de son piédestal. Ses trois fils aînés sont partagés entre un sentiment de révolte et de pitié pour ce père qui ne peut plus se réaliser dans le travail. II n'a pas réussi à se reconvertir dans une autre activité professionnelle : en effet, il voulait obtenir

8. Driss est un de ses fils âgé de 20 ans au moment de l'entretien. 
une formation pour devenir chauffeur de taxi, mais le médecin du travail lui a refusé cette demande.

D'après des témoignages recueillis auprès de médecins du travail en 1999 dans le cadre d'une enquête européenne ${ }^{9}$, la situation des travailleurs immigrés accidentés souhaitant bénéficier d'un reclassement professionnel reste aussi bloquée. C'est toujours dans le secteur du bâtiment que les risques d'accident du travail sont les plus élevés ${ }^{10}$. Pour Yves, médecin du travail, " quand un maçon immigré, sans bagage scolaire, quelquefois analphabète, ne peut plus travailler dans le bâtiment, on n'a plus besoin de lui. Que va-t-on lui faire? C'est là que démarre le rouleau compresseur de l'exclusion. L'une des principales limites de la COTOREP ${ }^{11}$, c'est l'analphabétisme. Les personnes analphabètes ne sont pas prises dans un centre de reconversion professionnelle parce que l'on considère qu'il faut avoir été scolarisé au moins un minimum pour suivre une formation 11.

Beaucoup de travailleurs immigrés n'ont pas eu de droit à des formations (par exemple d'apprentissage du français); ils n'y ont pas non plus droit après avoir subi un accident du travail. Ne s'agit-il pas là d'une injustice grave? Sans doute peut-on en partie l'expliquer par la volonté du Mouvement des entreprises de France (MEDEF), en charge, avec la Sécurité sociale, des accidents du travail et des maladies professionnelles, d'éviter les dépenses "inconsidérées".

Au cours de l'enquête menée auprès de la famille Nour, nous nous sommes aperçue que les enfants d'Amin ne savaient pas que leur père s'était vu refuser la possibilité de se reconvertir comme chauffeur de taxi. II avait choisi de ne pas le leur raconter. C'est pendant l'enquête qu'il a expliqué devant certains de ses enfants qu'il ne voulait pas que ceuxci pensent qu'il avait été traité de manière injuste. On retrouve ici son souci de ne pas attiser leur sentiment de révolte contre la société française. Cette information aurait peut-être permis à ses fils de prendre conscience qu'il n'avait pas accepté de manière soumise de ne plus pouvoir exercer une profession, car il avait monté un dossier pour obtenir

9. Témoignages recueillis par David Rohi, doctorant à l'EHESS. Cf. BERTAUX, Daniel et al., Entre intégration et exclusion. Une étude comparative des dynamiques locales de précarisation et de résistance à l'exclusion. recherche financée par l'Union européenne, programme TSER sur l'exclusion sociale, 1998-2001, $85 \mathrm{p}$.

10. Après une baisse du nombre d'accidents du travail jusqu'en 1992, on assiste à une hausse de près de $10 \%$ des accidents mortels au niveau national et de maladies professionnelles ayant entraîné la mort. C'est dans le secteur du bâtiment et des travaux publics que cette aggravation est la plus forte. Voir le dossier "Bilan 1998 des conditions de travail", Liaisons sociales, n' 12858, 26 février 1999.

11. Commission technique d'orientation et de reclassement professionnel. 
ce reclassement professionnel. Au cours de son entretien il a sorti de "l'armoire à papiers" sa demande ainsi que les réponses du médecin et de la COTOREP. Mais seule Leïla, sa fille aînée, qui l'avait aidé à constituer le dossier présenté à la COTOREP, était jusque-là au courant.

Monsieur Nour et sa femme Diamila se servent de leurs souvenirs d'enfance au Maroc et de leurs expériences vécues en France pour aider leurs enfants à se construire ce que nous appelons des ressources subjectives. Ce sont des "outils d'expériences" forgés à travers une réflexivité sur une mémoire familiale qui, d'une part, aident à se construire une éducation morale (apprentissage du courage et de l'effort...) et un système de valeurs et, d'autre part, permettent d'établir une distance (maîtrise de soi et apprentissage de la patience) face aux contraintes quotidiennes imposées par la précarité et particulièrement par la difficulté de trouver un emploi.

Nous avons pu constater que cette stratégie éducative, qui utilise la transmission aux enfants des expériences personnelles comme outil de socialisation, est mise en œuvre par nombre d'autres familles immigrées que nous avons rencontrées dans différents quartiers de France. Mais d'autres choisissent, au contraire, de taire leur histoire. Ils pensent, d'une part, que cette histoire n'est pas légitime dans l'espace public français et, d'autre part, que pour aider leurs fils et leurs filles à s'intégrer ceux-ci doivent laisser de côté et même effacer le passé familial. Venant de pays ayant connu la colonisation française et de zones rurales pauvres, ils ont le sentiment que leur origine nationale et de classe les dévalorise et peut constituer un obstacle sur le parcours d'intégration de leurs enfants. Ce parcours passé peut aussi être objet d'une souffrance silencieuse qui est transmise sans que les enfants en comprennent tovjours le sens.

Les effets de cette méconnaissance se retrouvent par exemple dans le témoignage d'un enseignant de $C M 2$ de l'école Romain-Rolland à Stains (Seine-Saint-Denis), dans la banlieve parisienne : " Ces enfants sont laissés dans l'ignorance de ce que sont leurs parents, donc de ce qu'ils sont euxmêmes. Et on s'étonne ensuite de leurs faibles capacités intellectuelles. Pourtant, ils sont intelligents, capables d'établir des liens, de réfléchir, de s'étonner. N'est-ce pas le silence de leurs parents, chargé de souffrance, qui les empêche d'apprendre, qui les rend sourds au reste, comme s'ils n'entendaient que cela? Pris dans un malheur non dit, à peine pensé, ils vivent une relégation dont ils ne pourront sortir pour s'ouvrir au monde qu'en nommant et en com- 
prenant les tenants et les aboutissants de ce malheur qui les possède sans avoir jamais été reconnu ni partagé $11^{12}$.

\section{Le primat de la langue}

Les familles immigrées sont généralement installées dans des quartiers qui, depuis le début des années 1980, sont des objets de "politiques de la ville". Cela met ces familles en contact avec beaucoup de professionnels du social. C'est dans ce contexte que le ressenti de honte déjà évoqué - et que certains parents éprouvent - est parfois alimenté par des travailleurs sociaux. Ils/elles ont été les premier(ère)s Français(es) que ces mères de famille ont rencontré(e)s à leur arrivée en France.

Yeza Boulahbel-Villac a montré que, pour les femmes algériennes ayant émigré en France, les travailleurs sociaux sont la principale source d'information et d'orientation culturelle ${ }^{13}$. Elle insiste sur le fait que ceux-ci tentent parfois de leur imposer un mode de vie et un fonctionnement familial fondés sur la "supériorité" de la langue et de la culture françaises. L'argument utilisé est celui qui consiste à "I favoriser la réussite scolaire des enfants 1 . Or les parents qui appliquent ce principe sont en grande difficulté : ils n'arrivent pas à transmettre leur propre langue; ils parlent un langage qui n'est pas le leur, en mauvais français. Ils éprouvent des difficultés à se faire comprendre et donc à communiquer en profondeur avec leurs enfants.

La question du bilinguisme dans les familles émigrées/immigrées est complexe et ne date pas d'hier. Ce qu'elle met en jeu est bien entendu considérable. Les débats d'experts continuent sur les effets de telle ou telle attitude parentale : encourager le bilinguisme ou le recours systématique au français pour accélérer l'intégration des enfants? Leur avis est partagé. Selon certains, en effet, la richesse des échanges verbaux peut souffrir du recours à une langue que la mère parle à peine, ce qui

12. VANTSES, Martine ; REGGIOS, Feya, "On leur a volé leur âme en les privant de leurs racines. Raconte-moi ton histoire". Entretien avec un enseignant d'une classe de CM2 de l'école RomainRolland à Stains (93), Nouvel Observateur, $n^{\circ} 2144,8$ décembre 2005. Au cours d'une enquête menée dans un quartier de Nantes entre 1989 et 1992 auprès de familles immigrées, nous avons pu constater une différence importante entre les parcours d'insertion des enfants devenus adultes. La connaissance de l'histoire familiale était un facteur déterminant pour leur réussite. C'était ce qui leur donnait le sens, la patience et le courage de persévérer dans leur quête d'un emploi. Voir DELCROIX, Catherine, "Discrédit et action collective. La lutte d'une association de pères musulmans", in : COSSÉE, Claire ; LADA, Emmanuelle ; RIGONI, Isabelle (sous la direction de), Faire figure d'étranger : regards croisés sur la production de l'altérité, Paris : Éd. Armand Colin, 2004, pp. 191-210.

13. Cf. BOULAHBEL-VILLAC, Yeza, "L'intégration au féminin des Algériennes en France", Espaces et Familles, $\mathrm{n}^{\circ}$ 16, mai 1991, $103 \mathrm{p}$. 
peut entraver le développement d'un bon rapport aux savoirs scolaires et d'un bon apprentissage de la prise de parole en public.

Les services de la Protection maternelle et infantile (PMI) sont aussi un endroit où l'on aborde la question de la langue qu'il est bon de parler avec ses enfants. Là aussi on encourage les parents à s'adresser en français à leur progéniture. Diamila, la mère, en témoigne : " On $m$ 'a dit : "Si tu ne comprends pas des choses, il faut parler en français à tes enfants. Ils vont f'expliquer, te l'apprendre". En plus, à la PMI, ils m'ont proposé d'apprendre l'alphabet français une heure par semaine ; mais je n'ai pas le temps avec mes huit enfants. En plus, je pense que c'est bien de parler le berbère avec mes enfants pour qu'ils puissent parler avec leurs grands-parents. Donc, si vous voulez, moi je parle trois langues : le berbère, le français et l'arabe avec d'autres femmes à la consultation II.

À travers le témoignage de Diamila se pose la question de la légitimité dans la société française de langues comme l'arabe ou le berbère, porteuses d'un bagage culturel et de richesses qui dépassent les frontières de la France et du Maghreb. Comme pour l'initiation à l'histoire coloniale, il existe vis-à-vis de l'apprentissage des langues utilisées par les anciens colonisés un préjugé négatif. On peut, par exemple, entendre un professeur à l'école ou un agent de la poste dire, après avoir entendu une mère et une fille se parler en arabe ou en berbère dans ces lieux publics : "Celles-là ne font vraiment pas d'effort... elles ne veulent pas s'intégrer! Qu'est-ce qu'elles cachent? II. Selon une telle logique, les enfants de familles ayant émigré du Maghreb les plus susceptibles d'être traités comme des citoyens français à part entière seraient ceux qui ont accepté de ne pas apprendre ni parler leur langue maternelle.

Diamila nous fait aussi comprendre que pour que les "jeunes des cités" se construisent une estime de soi, il faut qu'ils puissent se situer dans une continuité familiale, générationnelle. Mais, par ses pratiques éducatives, elle nous laisse voir qu'ils doivent aussi être initiés à l'histoire et à cette mémoire historique partagée, mais souvent occultée, qui relie les parents, les enfants eux-mêmes et la France.

\section{Transmission de la mémoire historique}

À l'automne 2005, avant les émeutes, nous participons à une réunion dans l'immeuble des Nour qui a pour objectif de regarder une longue émission télévisée consacrée au $60^{\circ}$ anniversaire du procès de Nuremberg. 
II y a dans le salon marocain des Nour des parents et des enfants de six familles voisines (soit une vingtaine de personnes) venus partager avec eux ce moment qui sera suivi d'une discussion. Cette rencontre est organisée dans un contexte politique national et local très tendu.

Cette année-là était, comme le souligne Pascal Blanchard, l'année II qui marque un tournant dans l'histoire de notre pays. On a l'impression que la "cocotte-minute", trop longtemps verrouillée, est en passe de nous éclater au visage. La montée des revendications communautaires, la loi du 23 février 2005 enjoignant les professeurs d'histoire à donner une lecture "positive" de la colonisation, la crise des banlieues, l'utilisation de la loi de 1955 sur l'état d'urgence pour réprimer les violences des banlieues... sont autant de signes de cette explosion ${ }^{14}{ }^{14}$.

Dans le quartier de la famille Nour ${ }^{15}$, qui est l'objet de la "politique de la ville", des incidents venaient d'éclater entre jeunes musulmans et juifs à propos de la deuxième Intifada et de la remise en cause de la véracité de la Shoah. Comme c'est la pratique dans cette cité, ces événements ont conduit à la formation d'une commission constituée de parents volontaires, d'enseignants et de professionnels du travail social chargée de proposer des actions susceptibles de faire baisser la tension. C'est ainsi qu'il a été décidé de consacrer une partie du cours d'histoire des collégiens du quartier à l'étude de la Shoah et au rôle joué par la France pendant la période 1940-1945. Les élèves ont visionné le film Nuit et brouillard ${ }^{16}$, et à cette occasion il a été demandé aux parents de relayer l'école en donnant la parole aux enfants pour qu'ils puissent exprimer leurs émotions et poser des questions.

C'est dans ce contexte que les Nour ont pris l'initiative de proposer à leurs voisins de venir chez eux regarder l'émission sur le procès de Nuremberg. Ils se sont décidés à organiser cette réunion après avoir vu leur fille Fatiha, âgée à l'époque de 14 ans, revenir en pleurs à la maison après avoir vu le film Nuit et brouillard. Fatiha nous a confié un peu plus tard : " Quand j'ai vu ces femmes et ces enfants morts et au bord de la mort,

14. Entretien mené par Sylvain Courage, Aude Lancelin, Elsa Vigoureux avec I'historien Pascal Blanchard, "Non à la guerre des mémoires", Le Nouvel Observateur, 8 décembre 2005. Voir aussi BLANCHARD, Pascal ; BANCEL, Nicolas ; LEMAIRE, Sandrine (sous la direction de), La fracture coloniale : la société française au prisme de l'héritage colonial, Paris : Éd. La Découverte, 2005, 310 p.

15. Le nom du quartier n'est pas donné car nous leur avons promis l'anonymat.

16. Réalisé en 1955, dix ans après la fin de la Deuxième Guerre mondiale, ce film donne les moyens de mettre une distance nécessaire à une lecture plus sereine de l'histoire. II est le premier à faire barrage à une éventuelle avancée du négationnisme, ainsi qu'à donner un avertissement sur les risques que présenterait une banalisation, voire le retour en Europe de l'antisémitisme, du racisme ou encore du totalitarisme. 
je me suis mise à pleurer, je transpirais. C'était pas possible que des juifs qui sont si forts et puissants aient été traités comme ça, tués ! C'est du racisme, je ne pensais pas qu'eux aussi en ont été victimes 11.

Si les Nour ont eu cette idée c'est aussi parce qu'ils sont particulièrement sensibles au fait qu'il y ait une bonne entente entre juifs et musulmans. Depuis l'enfance, Amin a comme meilleur ami un juif marocain, et dans le quartier Diamila et lui développent les échanges entre habitants juifs et musulmans.

Regarder des émissions d'actualité et notamment celles qui portent sur l'histoire est une pratique fréquente chez les Nour. Nous l'avons également observé chez d'autres familles modestes qui considèrent la télévision comme un outil éducatif particulièrement important quand on n'a pas les moyens culturels et économiques d'avoir accès autrement à l'information et à la connaissance. La parabole a intensifié ce phénomène en augmentant le nombre de chaînes accessibles.

Diamila nous a dit à plusieurs reprises : "C'est important de voir ensemble avec les enfants des émissions historiques et politiques car on peut parler... ils sauront plus de choses, ils réussiront mieux à l'école. Eux, ils sont français, voir les débats politiques, ça les fait être des citoyens $11^{17}$. On voit à travers les propos de Djamila que dans l'effort de transmission parental, une place est réservée à la discussion sur la mémoire historique, les parents ayant le souci de trouver des moyens de communiquer avec leurs enfants, souci d'autant plus marqué par la volonté d'atténuer les effets négatifs sur l'estime de soi du racisme et des situations de discrimination vécues par leurs enfants ${ }^{18}$.

Après l'émission sur le procès de Nuremberg, la première remarque du débat a porté sur ces juifs martyrisés qui avaient obtenu justice. Un garçon d'environ 18 ans a tout de suite ajouté : " Eux au moins, on les considère comme des victimes... c'est pas comme nos parents qui ont tellement souffert de la colonisation et qui sont méprisés! Et nous, on n'est pas des vrais Français, on n'a pas d'avenir comme Arabes II. La discussion s'est engagée ainsi sur le racisme et son interactivité ${ }^{19}$.

17. Les huit enfants Nour sont français. Leurs parents ont demandé la nationalité française, sans succès.

18. Cf. DELCROIX, Catherine, "Entre volonté de s'en sortir et discrimination : une trajectoire éclairante", Nouvelles Questions Féministes, vol. 26, n 3, 2007, pp. 82-99.

19. L'interactivité du racisme veut dire que des jeunes peuvent être dans certaines circonstances victimes et dans d'autres agresseurs. Face au racisme plusieurs réactions sont possibles ; elles varient d'une personne à l'autre et aussi dans le temps chez une même personne. Une première attitude conduit à l'isolement et au repli sur soi. Elle peut dans des circonstances extrêmes

Vol. 21, $n^{\circ}$ I23-I24 mai-août 2009 
Les propos de ce garçon correspondent au fait que $40 \%$ de jeunes hommes français originaires du Maghreb se déclarent discriminés, contre $29 \%$ des jeunes femmes ${ }^{20}$. Comme le soulignent dans leur ouvrage Nacira Guénif-Souilamas et Éric Macé, les préjugés envers les jeunes d'origine maghrébine sont bien plus intenses à l'égard des garçons que des filles ${ }^{21}$ et reprennent des représentations du discours colonial sur "I'homme musulman", "I représentant d'un islam actif, masculin, et séditieux $1 \|^{22}$. Ces stéréotypes dont leurs pères ont été victimes donnent une définition de "I l'Autre " (le garçon arabe) comme "l le subordonné culturellement différent ${ }^{23}$.

Ces propos ont déclenché des réponses chez les adultes qui, semble-til, ont parlé pour la première fois de leurs expériences du passé colonial. Ainsi, un des parents présents (âgé d'environ 65 ans), originaire d'Algérie, a raconté : "I C'est vrai, mon père avait une ferme, et un jour des militaires français nous ont chassés. La ferme a été donnée à un colon. Pour le mettre dehors on a traité mon père de sale musulman incapable d'exploiter sa terre et de mari violent. L'Algérie a gagné son indépendance. C'est du passé. C'est plus la France d'hier. Il y a des Français qui reconnaissent que la colonisation était injuste. II ne faut pas rester fixé sur le passé. II faut avancer, ne pas faire monter la violence. II faut expliquer, être patient. La France a de bons côtés, il faut saisir les opportunités 11 . La discussion est restée dans une impasse parce que le garçon et d'autres jeunes lui ont répondu qu'il n'était pas assez rancunier et qu'il était beaucoup trop soumis face aux Français. II faut ajouter que pour certains jeunes présents, la télévision n'est qu'un outil de propagande, qui sert à victimiser les juifs et à stigmatiser les Arabes et les musulmans 24 .

amener à l'autodestruction et à la dévalorisation. Une seconde attitude conduit à l'agressivité et à la violence et, enfin, une troisième est orientée sur l'ouverture aux autres, l'autocontrôle, la déconstruction des préjugés et, quand l'injustice est trop flagrante, elle passe par le dépôt d'une plainte devant la justice.

20. Cf. ROULLEAU-BERGER, Laurence, "Insertions segmentées, travail et discriminations de femmes migrantes et de leurs filles", in : ROULLEAU-BERGER, Laurence ; LANQUETIN, Marie-Thérèse (sous la direction de), Femmes d'origine étrangère : travail, accès à l'emploi, discriminations de genre, Paris : La Documentation française, 2004, pp. 13-70 (voir p. 54).

21. Cf. GUÉNIF-SOUILAMAS, Nacira; MACÉ, Éric, Les féministes et le garçon arabe, La Tour-d'Aigues : Éd. de L'Aube, 2004, $106 \mathrm{p}$.

22. CLANCY-SMITH, Julia, "Le regard colonial : islam, genre et identités dans la fabrication de l'Algérie française, 1830-1962", Nouvelles Questions Féministes, vol. 25, n¹, 2006, dossier "Sexisme et racisme : le cas français", pp. 25-40 (cf. p. 25).

23. Ibidem.

24. Les propos de ces jeunes nous montrent la nécessité de laisser plus de place aux autres mémoires blessées, même si la mémoire de la Shoah est devenue la mémoire mondialisée et partagée par 
Éric Marlière signale aussi, à propos d'une recherche qu'il a menée sur les jeunes d'une cité de la Seine-Saint-Denis, que la réception des programmes de télévision s'avère problématique pour la plupart d'entre eux. Même pour ceux qui travaillent et qui ne la regardent plus parce qu'ils s'estiment stigmatisés dans les médias, la télévision est toujours présente dans leur quotidien, qu'ils le veuillent ou non : " De qui se moquet-on? La télévision m'écœure. On te montre un Arabe fanatique dans les pays du Golfe ou un Maghrébin des banlieues sauvageon. Ça suffit! Je n'ai plus de télé chez moi! Mais maintenant c'est mes collègues qui me posent des questions quand il y a un attentat en Irak ou en Palestine [...]. Ça devient pesant à force ! " [K., 31 ans, "galérien", salarié, célibataire $]^{25}$.

Le point de vue de certains jeunes présents dez les Nour sur la télévision est donc en opposition avec celui de leurs parents, ce qui rend la communication intergénérationnelle difficile. Certains jeunes continuent à ne pas croire à la réalité de la Shoah.

Une autre remarque a été faite par une jeune fille de 16 ans : " Pourquoi ces juifs qui ont été dans le passé si maltraités par des Allemands et des Européens sont-ils devenus si violents avec les Palestiniens? II. Question qui a eu comme réponse de la part des parents : "I Attention à ne pas confondre les juifs et les Israéliens ! Comme Arabes et eux comme juifs, nous sommes tristes de ce terrible conflit, mais nous ne sommes pas là-bas; nous ne pouvons rien faire. C'est pas la peine de commencer une autre dispute II.

L'ensemble de la discussion montre que les parents seuls ne peuvent pas trouver les moyens de transmettre des informations sur l'histoire collective. Ils sont obligés de mettre en œuvre une action qui les amène à coopérer avec les enseignants pour éviter ce que Pascal Blanchard appelle "l la guerre des mémoires ". Ce débat participe à rendre légitime non seulement l'histoire de la Shoah, mais aussi l'histoire de leurs familles, leur culture d'origine, l'histoire de la colonisation et de la fracture coloniale. des mémoires dans le monde" (dossier), Hermès, $n^{\circ}$ 52, 2008, pp. 13-22.

25. MARLIĖRE, Éric, "Le sentiment d'injustice chez les jeunes d'une cité HLM", in : "Pratiques éducatives et jeunes en foyer" (dossier), Sociétés et jeunesses en difficulté, $\mathrm{n}^{\circ}$ 2, automne 2006, pp. 1-22. 


\section{En guise de conclusion}

Les transmissions intergénérationnelles impliquent un très gros effort de travail sur les événements biographiques et historiques. Certains parents choisissent le silence, leurs enfants sont alors livrés à eux-mêmes. C'est à eux que revient le fait de se construire des repères d'autant plus que la société française ne parle pas et ne légitime pas les rapports de la France avec les pays du Maghreb. Souvenons-nous du fait que l'histoire de l'immigration n'est devenue une discipline universitaire qu'en $1988^{26}$ et que l'histoire coloniale en France est encore fortement taboue malgré le remarquable travail de nombreux chercheurs ${ }^{27}$.

Pour les familles qui, comme la famille Nour, ont choisi de développer chez leurs enfants des ressources subjectives, c'est-à-dire des outils d'expérience $^{28}$, il ne s'agit pas seulement de raconter une histoire familiale la plus complète possible. Il y a un travail de sélection qui se fonde sur la volonté d'aider les enfants à se socialiser en construisant une estime de soi. Par cette stratégie éducative, les parents tentent de les préparer à affronter les risques du racisme et de la précarité. Finalement, en agissant ainsi, ces parents et ces jeunes communiquent, même si des conflits existent, notamment sur leur rapport à la France et aux possibilités que celle-ci offre à la nouvelle génération ; il n'y a donc pas de rupture intergénérationnelle. Malgré ce travail éducatif, imaginatif et parfois collectif, le changement de représentations réciproques n'est pas facile. C'est pourquoi rien n'est jamais gagné (ni rien n'est jamais perdu). La responsabilité des chercheurs et des politiques est engagée. Disqualifier ces parents des milieux populaires et immigrés ne permet pas de faire face à l'angoisse d'une génération en quête de réponses.

26. C'est l'ouvrage de NOIRIEL, Gérard, Le creuset français. Histoire de l'immigration, $X I X^{e}$ et $X X^{e}$ siècle, Paris : Éd. du Seuil, 1988, 438 p., qui a marqué le début de cette reconnaissance.

27. Cf. BHABHA, Homi, Les lieux de la culture. Une théorie postcoloniale, Paris: Éd. Payot, 2007 (1994), 414 p. ; SAID, Edward, Orientalism, New York : Vintage Books, 1994 (1979), 394 p. ; SAID, Edward, Culture et impérialisme. Paris : Éd. Fayard - Le Monde diplomatique, 2000, 555 p. ; STORA, Benjamin, La guerre des mémoires (entretiens), La Tour-d'Aigues : Éd. de l'Aube, 2007, 107 p. Voir aussi "Sexisme et racisme : les cas français" (dossier), Nouvelles Questions Féministes, vol. 25, n 1, 2006; "Sexisme, racisme et postcolonialisme" (dossier), Nouvelles Questions Féministes, vol. $25, \mathrm{n}^{\circ} 3,2006$.

28. Dans l'ensemble des travaux de recherche que nous avons menés sur les familles immigrées en situation de précarité depuis la fin des années 1980, nous avons eu l'occasion de rencontrer environ 200 familles réparties dans toute la France. Nous avons trouvé cet effort de communication en direction des enfants pour donner du sens aux expériences vécues par les parents dans la moitié de ces familles. 
